\title{
The construction quality control analysis of water supply \& drainage and hvac engineering
}

\author{
Zhiping Liu $^{1, \text { a }}$ \\ ${ }^{1}$ Jilin Agricultural University, Changchun, Jilin Province, China \\ a36670921@qq.com
}

\begin{abstract}
Keywords: Water supply \& drainage, Hvac engineering, Construction quality control, Analysis
\end{abstract}
\begin{abstract}
With the deepening of China's reform and opening up and the rapid development of national economy, further acceleration of urban development, urban population is increasing, water and electricity demand is bigger and bigger, grasp the water supply and drainage and hvac engineering construction technology, water supply and drainage and hvac engineering construction quality control is particularly important. In this paper, how to control the construction quality of water supply\&drainage and hvac engineering are studied.
\end{abstract}

\section{Introduction}

The acceleration of urbanization process, making the construction industry of China is a good development, but the current market is still a buyer's market, building industry, therefore, construction quality becomes one of the targets of the construction industry. Construction project construction process, construction of hvac effects associated with people's living comfort and its important, therefore, in the process of construction and installation, should control the key content, with specific project to design a premise, control the key construction content. Based on this, when the design, set out the requirements of construction plan, controls the hvac engineering design number, security project is using the rules of the masses [1].

\section{The quality problems of water supply \& drainage and hvac engineering}

The common problems in water supply \& drainage engineering. All quality prevention measures in construction process to do well, pipeline installation: the main quality common fault is run, run, drip, jams, poor passage, down the slope, support and sanitary ware coordinates and elevation of displacement, and poor thermal insulation and anti-corrosion [1].

1) Pipe threads joint leakage: required processing thread straight, smooth, no burr, constantly silk, not disorderly buckle, etc., pipe thread and fittings required between the correct use of packing, after the pipe installation requires rigor and strength test, carefully check the pipeline and the presence of defects such as cracks, voids, joint thread head are in good condition.

2) The pipeline leakage of flange joints: requirements flange joint surface parallel to each other, hole between the positive and flange gasket material and thickness and flange bolts used to comply with the design requirements and construction specification requirements.

3) The leakage of the welding joints: main defects of weld size does not conform to, bite meat, burn through, convex tumor, lack of penetration, porosity, crack, slag, in the process of construction must be in accordance with the construction of correct operation, groove and welding layer to clean up.

4) The leakage of pipeline undertake flange section: the interface should be carefully before cleaning nozzle, port along the flow direction of medium, socket reverse flow direction of medium, after the installation to be closed water test, shown as Fig.1. 


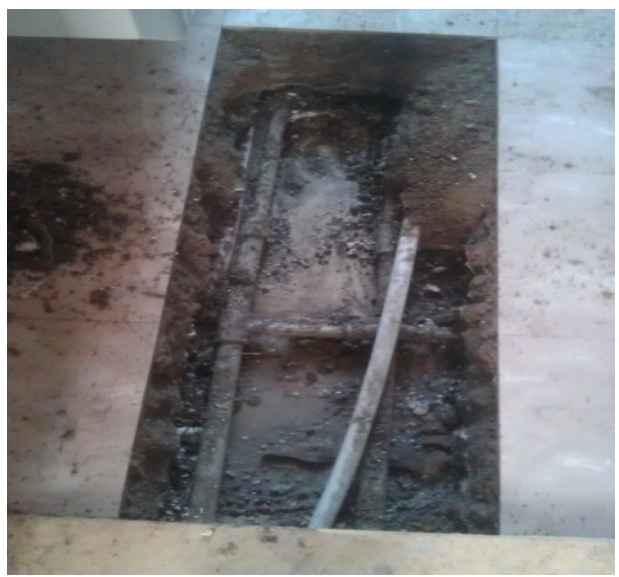

Fig. 1 Leaking pipes

5) Pipe support requirements: hanging bracket is chosen correctly in the construction process, and spacing of conform to the requirements of the specification and drawings, installation is firm, paton openings shall be strictly prohibited.

Valve packing requirements seal is good, the valve is not subject to any damage, gland to compaction, the playground is correct.

Poor water supply pipeline flow, water quality turbidity, water supply pipe installation should pay attention to clean up the pipeline internal dirt and sundries before and after the installation is to wash.

7) Drainage pipeline jam: drainage pipe installation should pay attention to clean up the internal stuff before, after the installation to drainage systems and the ball experiment was carried out.

8) Sanitary ware installation requirements: support required installing stability and strong, sanitary ware and metope is clingy and clean leveling.

9) Water pipe joint leakage on big toilet and: big toilet and water pipe joint of the rubber bowl with 14 copper wire stagger binding two way and screw down the big implement export and drainage pipe joint aperture, with a cream with 1:5 cement mixed with white putty or infill screening.

10) Floor drain water effect is bad: around the floor drain installation standard, correct reasonable slope, it is forbidden to down slope, perforated strainer $5 \mathrm{~mm}$ lower than the ground unity.

Pipe brush paint film have bubbles, lacquer layer have sank, rust paint film of the return flow phenomenon. Pipe brush paint to the first clean rust and dirt on the surface of the brush paint should pay attention to check, if there is any leakage brush carefully fill brush, flow falling part before the paint film is not completely dry with a shovel knife to remove.

Hvac engineering normal problems. Hvac equipment is the core part in hvac engineering, because the device involved in the process of selection and installation of the content are many, so it's easy to problems in the construction process. Embodied in the following ways [2]:

1) The hvac noise Para-normal in hvac air conditioning equipment. Excessive noise is one of the hvac system design problems are very common, with the development of modern science and technology, hvac noise Para-normal phenomenon got very big improvement. That will find the actual occurring in the construction of the noise is far higher than factory sample parameters. So during the design and construction, need to make clear a regulation also children's air conditioning noise figure. For noise of large air volume air conditioning equipment insulation treatment accordingly. Should be checked for all air conditioning equipment, electricity test before installation, a single found noise index does not meet the requirements of the contact manufacturer for replacement and adjustment, can be put into use only conform to the specifications.

2) Radiator slope is not reasonable. To be specific, the pipe in the material production measurement is not accurate, or the measurement deviation caused by deviation calculator. In order to avoid the occurrence of this phenomenon can take first installation radiator, then in the installation of main and riser, in accordance with the actual situation of hvac engineering. Main and riser jilt mouth location inaccuracy lies in the choice of construction in the process of the high precision measuring tools, not according to an analysis of the measurement data of the construction drawings. In accordance with the actual situation of the construction site under construction, the radiator coordinates inaccuracy due to the construction site search for radiator, the distance of the wall position nearly far. 
3) The valve installation is not correct. In the hvac engineering construction process, due to the use of the valve type is relatively more, in this case the phenomenon of installation errors are easy to happen. For example, set the exhaust valve in the position of the fire valve, or the reverse installation of valve, etc. Is common in some civil air defense engineering, the phenomenon of reverse installation manual valve, the main reason is confused by the valve in the direction of the construction work personnel, or negligence in the process of installation.

4) Hvac engineering pipeline condensation dripping problem. Pipeline condensation water is a serious engineering problem, will cause the hvac work occurred in a single fault, the system will not run properly. In a pipeline condensation dripping for many reasons, is relatively complex, common are: the installation of a pipeline system problems, pipe heat preservation measures were in place, the interface of the system is not strict, and so on. Of the most important reason is the piping installation problem, in the early stage of the installation process not strictly carried out in accordance with the operating procedures, material quality and the system of management and control does not reach the designated position, no hydrostatic testing of the system will lead to condensation dripping water, such as shown in Fig.2.

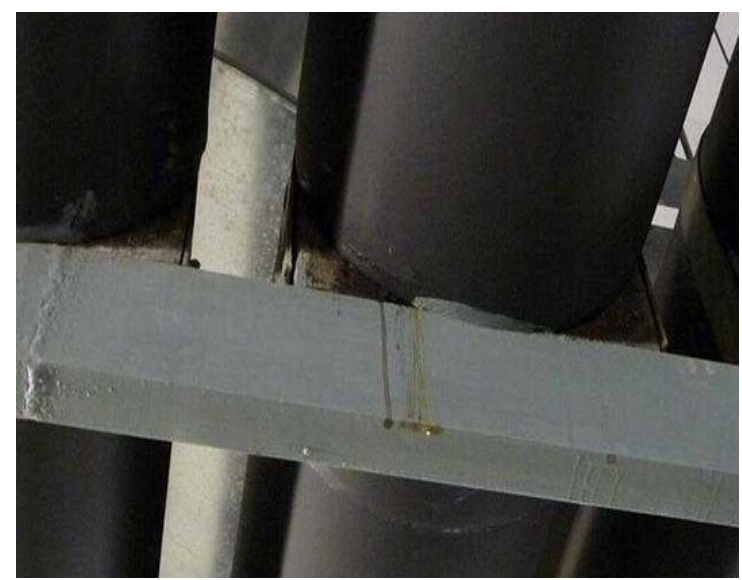

Fig. 2 Pipeline condensation dripping schematic diagram

\section{Water supply \& drainage and hvac engineering construction quality control measures}

Quality control, water supply and drainage installation project is an important part of the construction project, we will strictly according to quality acceptance criteria for acceptance of completed projects.Installation equipment, materials and finished products, semi-finished products of nameplate, model, specification, performance and construction technology of installation quality, must conform to the installation of relevant professional norms, standards, general projects allow deviation and checking method, but should comply with relevant regulations. Installation quality is an important factor directly affects the quality of construction projects, must formulate the feasible preventive measures before construction, the quality problems in the bud.

The quality control in advance. In engineering construction preparation stage, construction enterprises can't escrow "'" to the package, should insist to install the subcontract, project managers, construction personnel for the qualification of qualifications, adhere to the type of work in accordance with the related certificates [3]. The construction team training, screening.Before the construction of the professional construction team training, improve their quality consciousness and operation level, making the project quality standard.

Make quality controlIn the construction. Management personnel should be on site for all water supply and drainage project schedule, quality and safety of installation, uninterrupted complete tracking, coordination and inspection [3].In construction process, should be timely communication with the owner, coordinate related affairs, such as for examination and approval of construction organization, construction drawings came, find the solution of the problem in the process of construction and change of consultation, etc.

Focusing phase, usually including the basement, transition layer and standard head two layers of construction stage, due to the volume of flow is not enough continuity, the beginning of the 
construction in complex changes, it is easy to cause confusion and errors in construction of water supply and drainage, especially in the basement construction stage, almost all of the important equipment design and installation in the basement, equipment, pipeline, likely to touch, leak, wrong, lack of phenomenon, at this time the professional engineers should check the detail drawings, after verification must be strictly according to the diagram construction.

The final quality control. In strict accordance with the quality acceptance criteria for the acceptance of the construction [4].Building water supply and drainage installation engineering materials, electrical appliances, equipment and finished products, semi-finished products of the brand, model, specification, performance and construction technology of installation quality, must conform to the design and relevant professional norms and standards.

Strengthen the control of construction material accessories. Air conditioning equipment and pipelines and related materials and spare parts all affect the quality of the overall quality of construction quality and operation of hvac engineering. So be sure to do a good job in the quality control of equipment material to strengthen the inspection and acceptance of materials, equipment [5]. For the materials provided by the construction unit and construction unit itself purchase materials need to be professional inspection, inspection materials by technicians qualified certificate of equipment, the factory quality certificate, etc., have no related proof materials equipment are not allowed to enter the arena, at the same time, check the production date of materials, equipment, model, specification, quantity, etc is consistent with construction requirements [4]. Pipes, pipe fittings, radiators and valves and other projects in the extensive use of the original strict inspection of the factory quality certificate, eliminate no qualification of the original.Materials equipment shall be carried out in accordance with the name, type and specification and storage.Some materials need to undertake the construction intensity and rigor test, strictly to good quality.

A good job of drawing review. Construction drawings are important documents, review the drawings and engineering is an important work before construction, construction personnel should fully understand the main content of construction drawings, and according to the specific requirements of hvac engineering to check the drawings and specifications, check whether there is any omissions or potential problem, if the problems of drawing should be timely modifying drawings.Hvac engineering usually together with the civil engineering construction, therefore in the process, the blue prints.the design should pay attention to communication between the various type of work the construction technology of personnel and coordination, ensure the engineering safety [5].

Cooperate with all kinds of work. In the process of the rapid development of construction industry, more and more architectural function, based on this, is used to implement various functions increase gradually, the kinds of pipeline and equipment in addition, the home of condole top is relatively limited space, each line layout inside condole top, in case of improper coordination, there will be a line up and down wrong order or line positioning error [6].Therefore, construction of each work need close coordination, strengthen exchanges and communication between each other, need for coordination problem of unnecessary rework.

The construction process of quality control. Often occur during construction of heating main slope inappropriate phenomenon, there are two main reasons: one is because stent position and elevation of the pipe installation is no good control, the actual with the requirements for a larger deviation, lead to duct tape hog or dent.Pipe water vapor is easy to occur within the agglutinate phenomenon, and affect the operation of the whole system;Secondly because the process of installation of pipeline, pipeline itself without straightening, or is not installed in the working process of the empty hole in the wall plug slope.So, in order to let the installation incline more reasonable, need straightening pipe before installation, error reduction in minimum range, and then according to the slope and elevation to calculate the appropriate stent spacing, in strict accordance with the standard distance adjustment [6]. Pay attention to in the process of plug the hole in the wall, a small pipe could not enter, too, are likely to cause pipeline gradient change. 


\section{Summary}

To sum up, building water supply and drainage and hvac engineering is complicated system engineering, this is because, involved in the process of construction is more, if any one link problems, will make the overall quality is affected. Therefore, in the process of construction, need to be familiar with, understand and master the key construction technology at the same time corresponding measures are taken, further to control construction quality, to a certain extent, improve the overall quality of the buildings hvac engineering.

\section{References}

[1] Y.H. Li, Construction engineering quality management problems and their countermeasures, High-tech enterprises in China, 2009, vol.10, pp.16-19.

[2] Zh.H. Li, Building water supply and drainage engineering construction quality management, Information science and technology, 2007, vol.23, pp.61-64.

[3] M.G. Li, Introduction to water supply and drainage engineering construction management, Project management, 2007, vol.9, pp. 7-9.

[4] N.N. Ch, Hvac problems should be paid attention to in engineering construction, Promote the development of science and technology, 2009, vol,.5, pp.-168-172.

[5] Ch.L. Wang, Problems and measures in hvac engineering construction is analyzed, Explore science and technology, 2010, vol.9, pp.88-91.

[6] S. Han, Hvac construction and solving the problems in the method, Construction and building decoration, 2009, vol.1 pp. 122-126. 